\title{
Correlation of Clinical and Endoscopic Evaluation with PET-CT in Detecting Recurrence of Head and Neck Squamous Cell Carcinoma
}

\author{
Rambabu Mappuri, ${ }^{1}$ Ramakrishnan Narayanaswamy ${ }^{1}$
}

\section{Introduction}

ABSTRACT

The current study reports the correlation of clinical and endoscopic evaluation with 18 F-Fluorine Fluorodeoxyglucose Positron emission tomography/Computed tomography (18F-FDG PET/CT) findings in detecting the residual/recurrence tumor after definitive treatment in the follow up cases of squamous cell carcinoma head and neck.

\section{Materials and Methods}

A prospective comparative study carried out on 30 follow-up cancer patients after definitive primary treatment. All patients were evaluated by clinical examination, endoscopy and 18F-FDG PET/CT and these results correlated to detect residual/ recurrence. Suspicious lesion at primary site suggesting residual/recurrence was confirmed by gold standard investigation, histopathological examination (HPE).

Result

The age group mostly involved is 40-70 with male predominance. Predominant sites were oral cavity and larynx. 20 patients showed recurrence on clinical and endoscopic evaluation with 03 false positive results, 10 patients showed no recurrence with 01 false negative result. On PET/CT, 19 patients were showed recurrence with 01 false positive result, 11 patients showed no recurrence. PET/CT showed high sensitivity, specificity, with high NPV in detecting the recurrence of disease in the follow up period. Clinical and endoscopic evaluation also has high sensitivity, PPV and NPV.

Conclusion

It is recommended that $18 F-F D G$ PET/CT scan should be done in every case after primary treatment in addition to complete clinical and endoscopic evaluation and during follow-up period for early detection and management of residual/ recurrence. $\underline{\text { Keywords }}$

Positron Emission Tomography Computed Tomography; Fluorodeoxyglucose F18; Carcinoma, Squamous Cell; Neck; Head

$\mathrm{H}$ ead and Neck Squamous Cell Carcinoma (HNSCC) is a broad term that encompasses a heterogeneous group of malignancies originating from the nasal cavity, paranasal sinuses, oral cavity, pharynx and larynx. Head and Neck Squamous Cell Carcinoma (HNSCC) is the sixth most common type of cancer representing about $6 \%$ of all cancers and estimated 650,000 new cases and 350,000 cancer deaths worldwide every year.

Primary causes of HNSCC are tobacco and alcohol consumption and Human Papilloma virus (HPV) infection. HNSCC often spread to the lymph nodes in the neck, and this is often the first sign of the disease at the time of diagnosis. About two-third of the patients of HNSCC present with advanced stage disease. An approach focusing on combining history, physical examination, endoscopy and tissue sampling is the mainstay for diagnosis and staging of HNSCC. Clinical guidelines for HNSCC recommend different imaging approaches for each phase of disease. Imaging is now

1 - Department of ENT, Command Hospital(EC), Alipore

Road, Kolkata

Corresponding author:

Dr Ramakrishnan Narayanaswamy

email: drnramak5632@gmail.com 
an integral part of the work up; most common known modalities in clinical use are CT and MRI imaging. The role of 18F-FDG PET/CT has been evaluated in the initial staging as well as post treatment re-staging and monitoring response to definitive therapy of head and neck carcinomas. ${ }^{1,2}$ FDG PET/CT is extremely useful in the situations where anatomic imaging is equivocal and the disease cannot be assessed by direct visualization and detection of cervical lymph node metastases.

Over the last few years, lots of studies have been conducted evaluating the role of PET/CT in initial staging as well as follow up of locally advanced head and neck cancers. Most of the studies have shown a definitive value of PET/CT in detecting the residual or recurrence of tumor. However, very few studies published the importance of clinical and endoscopic evaluation along with PET/CT to improve the accuracy in diagnosing the residual/recurrence of HNSCC. There is paucity of data from developing countries including India where HNSCC is one of the commonest malignancies seen. Hence, there was envisaged to carry out one such study in government tertiary care set up of India.

This study was undertaken to determine the clinical impact of FDG PET/CT and clinical and endoscopic evaluation in early diagnosis of residual/recurrence in post treatment patients of HNSCC.

\section{Materials and Methods}

This is a prospective comparative study conducted at ENT Dept of a tertiary care centre in Eastern India between January 2016 to June 2017. Written and informed consent was taken from each patient undergoing the study. The study was carried out after the hospital ethical committee approved the study, since it involved taking biopsies in all cases, whether they had suspicion of recurrence or not.

For every patient included in the study, depending upon the primary site of malignancy, clinical examination, diagnostic nasal endoscopy, video laryngoscopy/fiberoptic laryngoscopy, panendoscopy, 18F-FDG PET/CT and biopsy from primary site were taken under appropriate anesthesia. False Positive and False negative rates of both clinical and endoscopic evaluation as well as that after PET-CT were assessed after histopathological examinations.

Inclusion criteria: Patients willing to be included in the study. All cases of squamous cell carcinoma of head and neck, biopsy proven HNSCC any-stage (according to American Joint Committee on Cancer staging) after primary treatment originating in the lip, oral cavity, oropharynx, nasopharynx, paranasal sinuses, hypopharynx, glottis, larynx, or supra-glottic region.

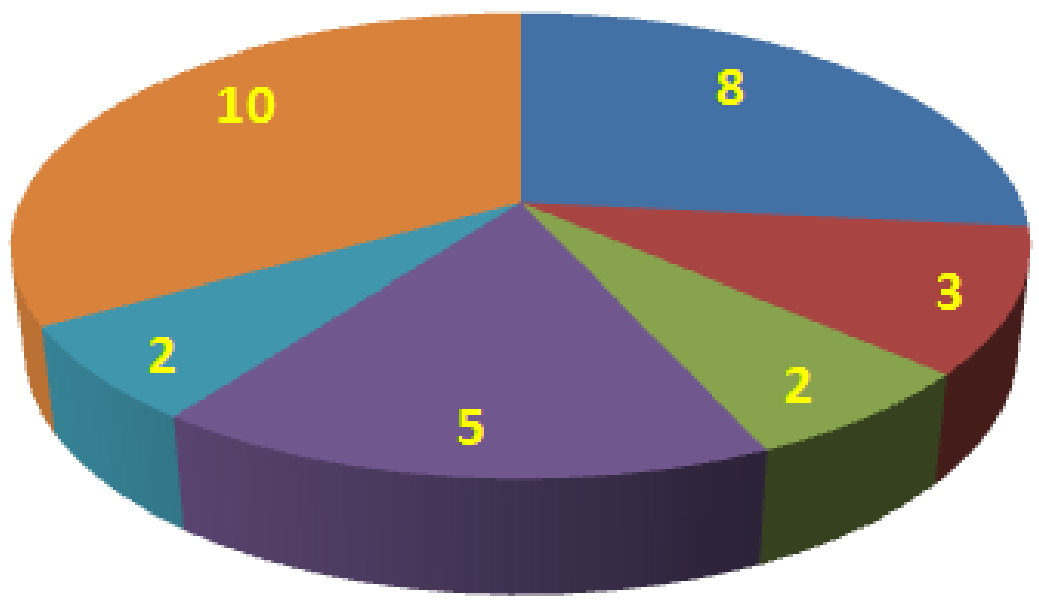

ORAL CAVITY

- NOSE\&PNS

NASOPHARYNX

- OROPHARYNX

- HYPOPHARYNX

- LARYNX

Fig.1. Anatomical site distribution 
Exclusion criteria: Primary cases before definitive treatment, carcinoma of unknown primary site, all nonsquamous cell carcinomas of head and neck, patients with metastatic disease, history of prior or concurrent second malignancy and follow up of missed cases were excluded from the study.

Categorical variables were expressed as number of patients and percentage of patients and compared across the groups using Pearson's Chi Square test for Independence of Attributes/ Fisher's Exact Test, as appropriate. Association of outcome of PET CT and Clinical Examination with HPE is captured using Sensitivity, Specificity, PPV, NPV and Diagnostic accuracy. The statistical software SPSS version 20 has been used for the analysis. An alpha level of 5\% has been taken, i.e. if any $\mathrm{p}$ value is less than 0.05 it has been considered as significant. If the calculated $p$-value is below the threshold chosen for statistical significance (usually the 0.10 , the 0.05 or 0.01 level), then the null hypothesis rejected in favour of alternative hypothesis.

Result
Total 30 patients were followed-up, 23 patients were male and 07 patients were female. Age distribution of all patients were between 26 years to 82 years. Maximum number of cases was in the age group of 51 to 60 years. Main bulk of cases was above 50 years. of age. Anatomical site distribution of lesions (Fig.1) shows that out of 30 cases, 08 cases were carcinoma oral cavity (26.67\%), 03 cases were carcinoma Nose and PNS (10\%), 02 cases of Nasopharyngeal carcinoma (6.67\%), 05 cases were carcinoma Oropharynx (16.67\%), 02 cases were carcinoma hypopharynx (6.67\%) and 10 cases were carcinoma larynx (33.33\%).

Distribution of recurrence based on evaluation: In 20 cases, clinical and endoscopic recurrence was found in only in 18 cases only, which was proven histopathologically. No evidence of recurrence was found in 10 cases. PET/CT showed recurrence in 19 cases, no recurrence in 11 cases. The accuracy of PET/ CT is $94.73 \%$, clinical and endoscopic evaluation is 90\% (Table I).

Diagnostic accuracy of PET/CT: The diagnostic accuracy of PET/CT is 96.67 with sensitivity,

Table I: Distribution of recurrence based on evaluation

\begin{tabular}{|c|c|c|c|c|}
\hline MODE OF EVALUATION & $\begin{array}{c}\text { RECURRENCE } \\
\text { PRESENT }\end{array}$ & $\begin{array}{c}\text { NO } \\
\text { RECURRENCE }\end{array}$ & $\begin{array}{c}\text { RECURRENCE } \\
\text { CONFIRMED BY } \\
\text { HPE }\end{array}$ & ACCURACY \\
\hline Clinical and Endoscopic & 20 & 10 & 18 & $90 \%$ \\
\hline PET/CT & 19 & 11 & 18 & $94.73 \%$ \\
\hline
\end{tabular}

Table II: Diagnostic accuracy of PET/CT

\begin{tabular}{|c|c|c|c|c|c|c|c|c|c|}
\hline 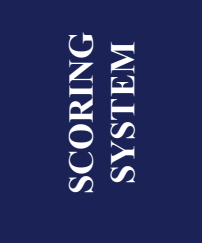 & $\hat{\theta}$ & Z & $\frac{\theta}{a}$ & $\mathbb{Z}$ & $\sum_{\vec{D}}^{E}$ & $\frac{\vec{b}}{\vec{U}}$ & ฉ & $\frac{8}{z}$ & 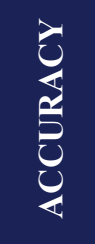 \\
\hline PET CT & 18 & 11 & 1 & $\mathbf{0}$ & 100 & 91.67 & 94.74 & 100 & 96.67 \\
\hline $\begin{array}{l}\text { Clinical and } \\
\text { Endoscopic }\end{array}$ & 17 & 9 & 3 & 1 & 94.44 & 75 & 85 & 90 & 86.67 \\
\hline
\end{tabular}




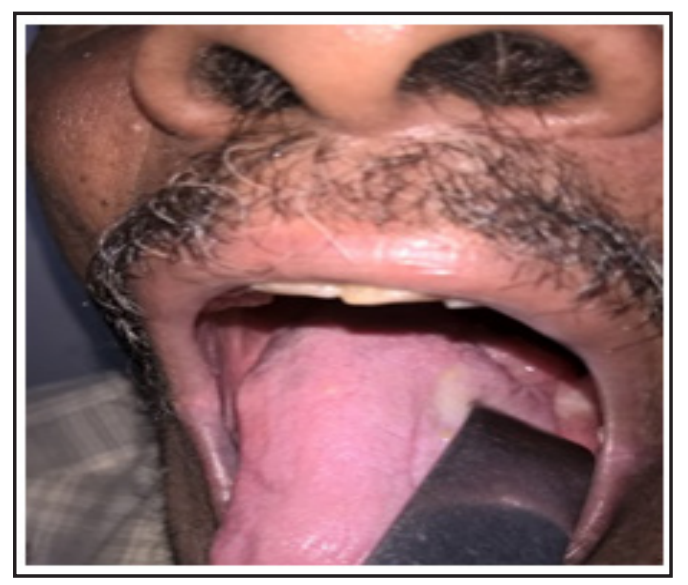

Fig. 2a. Tongue - no tumor seen but cervical lymph node was palpable

specificity; PPV and NPV were 100, 91.67, 94.74 and 100 respectively. There was 01 false positive case and no false negative cases (Table II). The diagnostic accuracy of clinical and endoscopic evaluation is 86.67 with sensitivity, specificity; PPV and NPV of 94.44, 75, 85 and 90. False positive cases were 03 and false negative cases 01 (Table II).

Significance of clinical and endoscopic examination and PET/CT: Clinical and endoscopic evaluation showed recurrence in 20 cases with no recurrence in 10 cases. After comparing the clinical findings with PET/ CT findings we found in 03 cases clinical findings were different with $\mathrm{PET} / \mathrm{CT}$.

Case 01-Case of carcinoma base of tongue, clinically no recurrence seen at primary site (Fig. 2a) but left level II lymph node palpable, 18F-FDG PET/CT showed FDG avid uptake (Fig. 2b), biopsy of that node showed

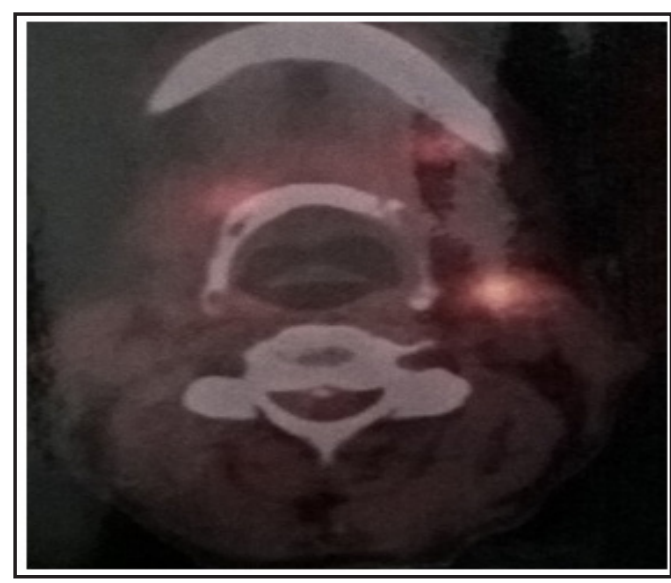

Fig. 2b. PET/CT- FDG avid uptake in level II cervical lymph node

inflammatory changes, so recurrence was ruled-out.

Case 02- Case of Nasopharyngeal carcinoma, on nasal endoscopy bulging seen on nasopharyngeal wall, PET/ CT showed no FDG uptake.

Case 03- Case of carcinoma oral cavity (right lower alveolus, post-surgery + CCRT) clinically operated area showed only edema, no tumor growth seen. PET/CT showed FDG avid uptake at same area and HPE reported as squamous cell carcinoma. The diagnostic value of clinical and endoscopic evaluation is significant with p-value of $<0.001$ (Table III).

$\mathrm{PET} / \mathrm{CT}$ showed recurrence in 19 cases, no recurrence in 11 cases, only in 18 cases recurrence was proven histopathologically. Due to inflammation PET/CT showed FDG avid uptake in left level II lymph node in a case of carcinoma base of tongue. Diagnostic value of $\mathrm{PET} / \mathrm{CT}$ is significant with $\mathrm{p}$-value of $<0.001$ (Table IV).

Table III: Significance of clinical examination

\begin{tabular}{|c|c|c|c|c|c|c|}
\hline & \multicolumn{2}{|c|}{ PET CT } & \multirow{2}{*}{ TOTAL } & \multirow{2}{*}{$\begin{array}{c}P \\
\text { VALUE }\end{array}$} & \multirow{2}{*}{ SIGNIFICANCE } \\
\hline & & NEGATIVE & POSITIVE & & & \\
\hline \multirow{2}{*}{$\begin{array}{l}\text { Clinical and } \\
\text { Endoscopic } \\
\text { examination }\end{array}$} & No Recurrence & $9(81.82)$ & $1(5.26)$ & $10(33.33)$ & \multirow{2}{*}{$<0.001$} & \multirow{2}{*}{ Significant } \\
\hline & Recurrence present & $3(18.18)$ & 17(94.74) & $20(66.67)$ & & \\
\hline \multicolumn{2}{|r|}{ Total } & $12(100)$ & $18(100)$ & $30(100)$ & & \\
\hline
\end{tabular}


Table IV: Significance of PET/CT

\begin{tabular}{|c|c|c|c|c|c|c|}
\hline & & \multicolumn{2}{|c|}{ PET CT } & \multirow{2}{*}{ TOTAL } & \multirow{2}{*}{$\begin{array}{c}\text { P } \\
\text { VALUE }\end{array}$} & \multirow{2}{*}{ SIGNIFICANCE } \\
\hline & & NEGATIVE & POSITIVE & & & \\
\hline \multirow{2}{*}{$\begin{array}{c}\text { CONFIRMATORY } \\
\text { HPE }\end{array}$} & Negative & 11(100) & $1(5.26)$ & $12(40)$ & \multirow{2}{*}{$<0.001$} & \multirow{2}{*}{ Significant } \\
\hline & Positive & $0(0)$ & 18(94.74) & $18(60)$ & & \\
\hline \multicolumn{2}{|c|}{ Total } & 11(100) & $19(100)$ & $30(100)$ & & \\
\hline
\end{tabular}

If patient shows recurrence both clinically and in PET/ CT carries high diagnostic value than either modality alone. Figs. 3a, 3b, 3c shows a case of Carcinoma Maxilla (left) operated with tumor recurrence seen clinically at flap site and in neck nodes and FDG avid uptake seen in both areas, which was confirmed by HPE.

\section{Discussion}

18F-FDG PET/CT has been used to detect recurrent disease in the head and neck cancer patients. It has also shown good accuracy in diagnosing asymptomatic and subclinical recurrences at the site of the treated primary disease, disease in reconstructed flaps and nodal recurrence post radiation. Recurrence in PET/ $\mathrm{CT}$ is visualised as enhancing lesion with intense FDG avidity. Risk of developing HNSCC also increases with age and the majority of HNSCCs occur in patients aged 50 years or over. The average age for smoking related HNSCC diagnosis is 60 years (median age: 63 years) whereas the average age for smokeless tobacco related HNSCC is 78 years. HNSCC is more common in men than in women and the ratios of Oral and Oropharyngeal SCC by gender are currently about 1.5:1 and 2.8:1, respectively. ${ }^{3,4}$

In this study, total 30 patients were followed up after primary treatment with male predominance. The proportion between male and female were 3.28:1. Major bulk of cases was above 50 years of age. The trends of age and sex of the patients were in close relation to other studies. Common modality of treatment in advanced cases of oropharynx, hypopharynx was CCRT and in
T1 lesions of larynx definitive RT was used as primary treatment. The ideal time to perform PET/CT in the follow up period should be 12 weeks of completion of CCRT to reduce false positive results.

Ryan et al. conducted a study to determine the diagnostic accuracy and the ideal timing of FDG PET in the post treatment surveillance of head and neck mucosal squamous cell carcinoma (HNSCC) on 118 post treatment PET scans at 12 weeks, who had undergone treatment for HNSCC and concluded that PET is effective in detecting distant metastases in the post treatment surveillance for HNSCC patients. ${ }^{5}$ A negative PET is highly reliable for all sites. However, a positive PET-CT in the head and neck region is unreliable because of a high false-positivity rate. PET-CT of the head and neck region has a statistically significant risk of a false-positive reading, when performed within 01 month of radiotherapy. ${ }^{5}$ Nelissen et al. showed that PET/CT carried out before 3 months had higher false negative results and higher false-positive rates. ${ }^{6}$ In our study, we conducted PET/CT after 12 weeks of primary treatment to reduce the false positive results. If patient shows recurrence both clinically and in PET/CT, it carries high diagnostic value than either modality alone.

Figs. 3a, 3b, 3c shows a operated case of Carcinoma Maxilla (left) with clinically tumor recurrence seen at flap site and neck nodes with FDG avid uptake seen in both areas, which was confirmed by HPE. Bundhit et al. reported sensitivity, specificity, PPV and NPV of PET/ $\mathrm{CT}$ for detection of residual primary tumor of $94 \%, 82 \%$, $75 \%$ and $95 \%$ respectively. ${ }^{7}$ Abgral et al. in a series of 91 patients with HNSCC who have finished their initial 


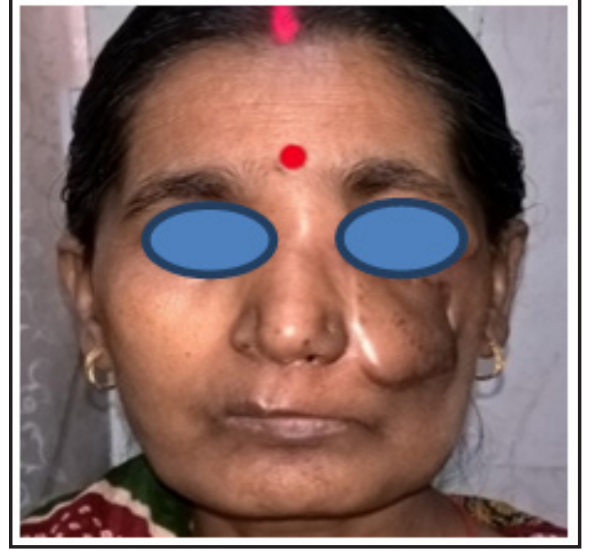

Fig. 3a. Flap involved by tumor in a case of Carcinoma Maxilla operated

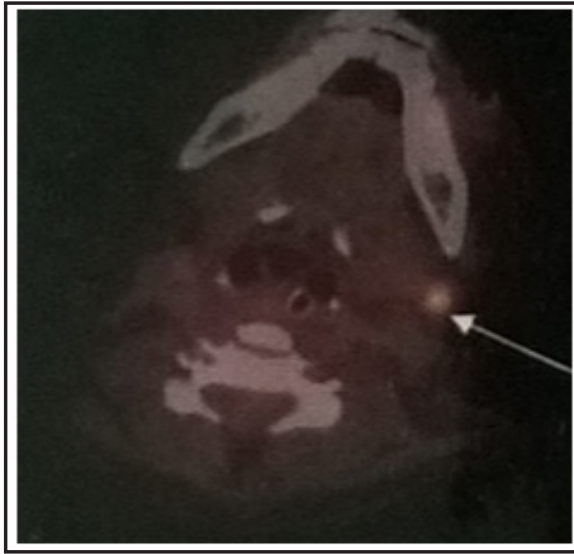

Fig. 3b. Cervical Level II lumph node showing FDG avid uptake

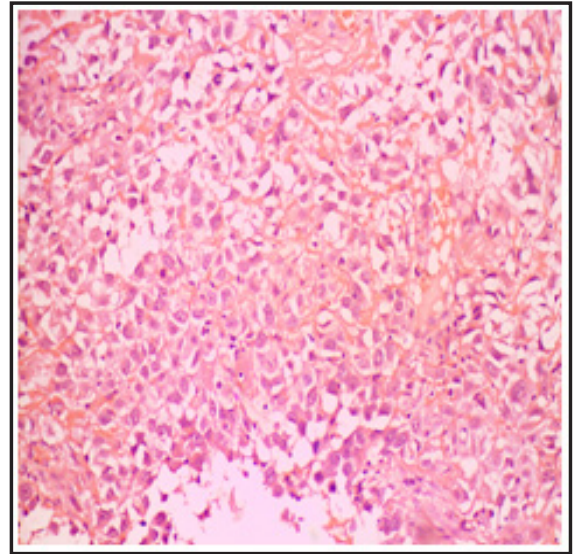

Fig. 3c. HPE confirms recurrence (SCC) (H and E, 200X) therapy reported sensitivity, specificity, PPV and NPV were $100 \%, 85 \%, 77 \%$ and $100 \%{ }^{8}$ Two recent studies also showed increased accuracy with delayed PET/CT performed approximately 4 months after treatment with NPVs reaching $100 \%{ }^{9,10}$ It is noted $18 \mathrm{~F}-\mathrm{FDG}$ PET/CT has high NPV, therefore negative results suggest absence of viable residual disease. ${ }^{7-10}$ In our study sensitivity, specificity, NPV and PPV of PET/CT was $100 \%$, $91.67 \%, 94.74 \%$ and $100 \%$. False positive results were mainly due to inflammatory changes and edema after radiotherapy.

Inflammatory changes in tissue may pick up FDG and showed as false positive results. The sensitivity and NPV of clinical and endoscopic evaluation were also high but not more than PET/CT. False positive results mainly due to distorted anatomy, inflammatory changes and edema after primary treatment. These results were in close relation to other studies results. Wang ${ }^{11}$ et al prospectively evaluated 44 restaging PET/CT between 12 and 17 weeks after radiotherapy completion, in the follow-up of 44 patients. Imaging data were compared with clinico-pathological outcomes, sensitivity, specificity, PPV and NPV was $100 \%, 98 \%, 92 \%$ and $100 \%$. In pooled analysis of over 2300 patients Gupta et al. reported a weighted mean $(95 \% \mathrm{CI}) \mathrm{NPV}$ of a single post-treatment PET/CT for the primary site at $95.1 \%$ (93.5-96.5\%). ${ }^{12}$ Clinical and endoscopic evaluation along with PET/CT carries more diagnostic accuracy than either modality alone in reducing the false positive cases.

\section{Conclusion}

This study was carried out with the objective of correlation of clinical and endoscopic evaluation with 18F-FDG PET-CT findings in detecting the recurrence or residual tumor after definitive treatment (chemotherapy or surgery or surgery and CCRT or CCRT) in the followup cases of squamous cell carcinoma head and neck. In the present study, all the cases were followed up after definitive primary treatment and underwent complete clinical and endoscopic evaluation and 18F-FDG PET/ CT. PET/CT showed high sensitivity, specificity, with high NPV in detecting the recurrence of disease in the follow up period. Clinical and endoscopic evaluation also has high sensitivity and NPV.

However, in detecting the recurrence- clinical and endoscopic evaluation along with PET/CT showed more accuracy than either modality alone in the follow up cases of HNSCC. In view of nearly $100 \%$ NPV of FDG PET-CT, it is recommended that every case after primary treatment should undergo complete clinical and endoscopic evaluation and 18F-FDG PET/CT scan during follow-up period for early detection of residual/ recurrent disease.

\section{References}

1. Abouzied MM, Fathala A, AI Muhaideb AI, AI Qahtani MH, Role of fluorodeoxyglucose-positron emission tomography in the evaluation of head and neck carcinoma. World J Nucl Med. $2017 ; 16: 257-65$ 
2. Cacicedo J, Navarro A, Del Hoyo O, Gomez-Iturriaga A, Alongi F, Medina JA, et al. Role of fluorine-18 fluorodeoxyglucose PET/CT in head and neck oncology: the point of view of the radiation oncologist. Br J Radiol. 2016; 89: 20160-217

3. Proia NK, Prszkiewicz GM, Nasca MA, Franke GE, Pauly JL. Smoking and smokeless tobacco-associated human buccal cell mutations and their association with oral cancer $-\mathrm{a}$ review. Epidemiol Biomarker Prev. 2006; 15: 1061-77

4. Hannah A, Scott AM, Tochon-Danguy H et al. Evaluation of 18F-FDG PET/CT with Histopathological correlation in the initial staging of head and neck cancer. Ann Surg. 2002; 236: 208-17

5. Ryan WR, Fee WE Jr, Le QT, et al. Positron-emission tomography for surveillance of head and neck cancer. Laryngoscope 2005; 115:645-50

6. Nelissen C, Sherriff J, Jones T, Guest P et al., The Role of Positron Emission Tomography/Computed Tomography Imaging in Head and Neck Cancer after Radical Chemoradiotherapy. Clin Oncol.(R Col Radiol.) 2017; 29 (11): 753-9

7. Bundhit T, Yu F, Kanard A, Miller F R. Role of 18-FDG PET/ $\mathrm{CT}$ in pre and post treatment evaluation in head and neck carcinoma. World J Radiol. 2014;6(5):177-91
8. Abgral R, Querellou S, Potard G, Le Duc-Pennec A, Marianovski $\mathrm{R}$, et al. Does 18F-FDG PET/CT improve the detection of post treatment recurrence of head and neck squamous cell carcinoma in patients negative for disease on clinical follow-up? J Nucl Med. 2009;50:24-9

9. Zundel MT, Michel MA, Schultz CJ, Maheshwari M, Wong SJ, Campbell $\mathrm{BH}$, et al. Comparison of physical examination and fluorodeoxyglucose positron emission tomography/computed tomography 4-6 months after radiotherapy to assess residual head-and-neck cancer. Int J Radiat Oncol Biol Phys. 2011; 81: e825-32

10. Slevin F, Subesinghe M, Ramasamy S, Sen M, Scarsbrook AF, Prestwich RJ. Assessment of outcomes with delayed (18)F-FDG PET-CT response assessment in head and neck squamous cell carcinoma. Br J Radiol. 2015; 88(1052):20140592

11. Wang YF, Liu RS, Chu PY, Chang FC, Tai SK, Tsai TL, et al. Positron emission tomography in surveillance of head and neck squamous cell carcinoma after de- finitive chemoradiotherapy. Head Neck 2009; 31: 442-51

12. Gupta T, Master Z, Kannan S, Agarwal JP et al. Diagnostic performance of post-treatment FDG PET or FDG PET/CT imaging in head and neck cancer: a systematic review and metaanalysis. Eur J Nucl Med Mol Imaging. 2011; 38(11):2083-95. 